\title{
Regular and non regular snore features as markers of SAHS
}

\author{
J. Mesquita, J.A. Fiz, J. Solà-Soler, Member IEEE, J. Morera, and R. Jané, Member IEEE
}

\begin{abstract}
Sleep Apnea-Hypopnea Syndrome (SAHS) diagnosis is still done with an overnight multi-channel polysomnography. Several efforts are being made to study profoundly the snore mechanism and discover how it can provide an opportunity to diagnose the disease. This work introduces the concept of regular snores, defined as the ones produced in consecutive respiratory cycles, since they are produced in a regular way, without interruptions. We applied 2 thresholds $\left(\mathrm{TH}_{\text {adaptive }}\right.$ and $\left.\mathrm{TH}_{\text {median }}\right)$ to the time interval between successive snores of 34 subjects in order to select regular snores from the whole all-night snore sequence. Afterwards, we studied the effectiveness that parameters, such as time interval between successive snores and the mean intensity of snores, have on distinguishing between different levels of SAHS severity (AHI (ApneaHypopnea Index) $<5 h^{-1}, \mathrm{AHI}<10 \mathrm{~h}^{-1}, \mathrm{AHI}<15 h^{-1}, \mathrm{AHI}<30 h^{-1}$ ). Results showed that $\boldsymbol{T H}_{\text {adaptive }}$ outperformed $\boldsymbol{T H}_{\text {median }}$ on selecting regular snores. Moreover, the outcome achieved with non-regular snores intensity features suggests that these carry key information on SAHS severity.
\end{abstract}

\section{INTRODUCTION}

NORING frequently accompanies SAHS (Sleep ApneaHypopnea Syndrome) and is universally recognized as one of its earliest symptom [1;2]. Hence, the most recent research in this field is focused on understanding this breathing disorder and proving that it can provide us the earliest opportunity to diagnose/screen this disease.

Several techniques such as sound intensity calculations, power spectrum analysis, feature extraction in time and frequency domains and attempts to model snore sounds are the latest efforts giving evidence that snoring carries information on SAHS [3-5]. Even though these latest works added auspicious information there seems to be a lack of emphasis on how the anatomical structure of the upper airways has effect on the time production of snores. Very few studies address the potential that sequential properties of snoring have on screening of SAHS[6].

In this work we introduce the concept of regular snores, as the ones produced in consecutive respiratory cycles, since they are produced in a regular way, without

This work was supported by the grant BES-2008-007617 and TEC2007-68076-C02-01 of the Ministerio de Ciencia e Innovación, from Spain.

J.Mesquita, J.Solà-Soler and R.Jané, are with Dept. ESAII, Universitat Politècnica de Catalunya (UPC), Institut de Bioenginyeria de Catalunya (IBEC) and CIBER de Bioengenieria, Biomateriales y Nanomedicina (CIBER-BBN) Baldiri Reixac, 4, Torre I, 9 floor, 08028, Barcelona, Spain (e-mails: jmgil@ibec.pcb.ub.es; jordi.sola@upc.edu;raimon.jane@upc.edu)

J.Morera is with Hospital Universitari Germans Trias i Pujol, Badalona (HUGTP), Spain josepmorera.germanstrias@gencat.net)

J.A.Fiz, is with Hospital de Navarra, IBEC, CIBER-BBN and HUGTP, Spain (e-mail: jafiz@msn.com) interruptions. For that we study the time interval between successive snores.

Not all time intervals between snores can be considered because they can either be: apnea episodes or periods of time where the subject is simply breathing (inspiration and exhalation events). For that reason, we applied 2 different thresholds to the time interval between successive snores in order to select the regular snores from 34 subjects. Our purpose is to study the characteristics of regular and non-regular snores and to uncover if their features enable to distinguish between different levels of SAHS severity subjects: AHI (ApneaHypopnea Index) $<5 \mathrm{~h}^{-1}, \mathrm{AHI}<10 \mathrm{~h}^{-1}, \mathrm{AHI}<15 \mathrm{~h}^{-1}$ and $\mathrm{AHI}<30 \mathrm{~h}^{-1}$. These four different levels are proposed by physicians and clinical experts as criteria for SAHS definition $[1 ; 7 ; 8]$.

\section{MATERIALS AND METHODS}

\section{A. Signal acquisition}

Snoring sound signals were acquired while full-night polysomnography was being performed on the sleep disorders laboratory of the Hospital Universitari Germans Trias $i$ Pujol in Badalona, Spain. Snoring sound was recorded with a unidirectional electric condenser microphone placed over the trachea at the level of the cricoid cartilage using an elastic band. The sound signal was amplified and filtered using second order Butterworth pass-band filter between 70 and $2000 \mathrm{~Hz}$ and digitized with a sampling frequency of $5000 \mathrm{~Hz}$ and a 12-bit analog/digital converter [5].

\section{B. Database}

The respiratory sound signal database consisted of 34 subjects ( 8 females and 26 males with age range of 37-72 years and Apnea-Hypopnea Index range of 3.7-109.9h ${ }^{-1}$ ) described in Table I.

TABLE I

CHARACTERISTICS OF THE SUBJECTS DATABASE

\begin{tabular}{cccccc}
\hline \hline & AHI & NSnores & Age & BMI & Number \\
\hline \hline m & 37.6 & 2190 & 50 & 28.5 & 34 \\
sd & 30.1 & 937 & 10 & 3.9 & $(8 \mathrm{~F} ; 26 \mathrm{M})$ \\
\hline AHI = Apnea-Hypoapnea Index, NSnores= total number of snores, \\
BMI = Body Mass Index, F = female, M=male, $\mathrm{m}=$ mean value, \\
sd=standard deviation
\end{tabular}




\section{Snore identification and Threshold application}

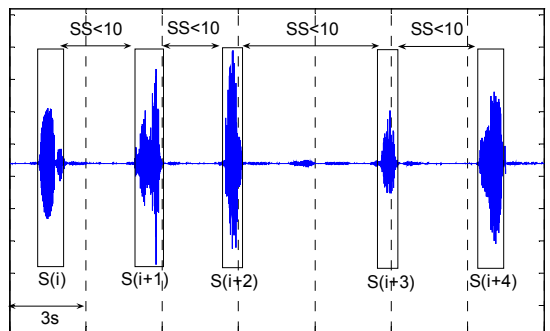

Fig. 1 Example of an excerpt of a snoring sound signal extracted from a subject from our database

Snoring episodes were identified by a proficiently trained and validated automatic detector and analyzer [5;9](Fig1).

Let $S S$ be the time interval between successive snores:

$$
\begin{gathered}
S S(i)=S_{\text {on }}(i)-S_{\text {on }}(i-1) \\
i=1, \ldots, \text { NSnores }
\end{gathered}
$$

where $S_{\text {on }}(i)$ is the onset of the detected $i$ th snore $S(i)$ and NSnores is the total number of detected snores.

Previous studies never offered a proper and decisive explanation for the fact that all time intervals between snores should be considered if they have less than 10 seconds [6;10]. The 10 second threshold $\left(T H_{10}\right)$ is based on the accepted convention that an air flow cessation that lasts more than 10 seconds is scored as an apnea[7].

Since we are interested in selecting regular snore episodes from all night (6 hours) sequences, we applied two different thresholds to the $S S(i)$ in order to identify consecutive snoring respiratory cycles.

The adaptive threshold, $T H_{\text {adaptive }}(i)$, is defined as follows:

$$
\begin{gathered}
T H_{\text {adaptive }}(i)= \begin{cases}\theta, & i<10 \\
A+B & , \text { otherwise }\end{cases} \\
A=(1-\delta) \frac{\sum_{10}^{i} S S(i-1) \mathrm{H}\left[T H_{\text {adaptive }}(i-1)-S S(i-1)\right]}{\sum_{10}^{i} \mathrm{H}\left[T H_{\text {adaptive }}(i-1)-S S(i-1)\right]-1} \\
B=\delta \frac{\sum_{10}^{i} S S(i-1) \mathrm{H}\left[T H_{\text {adpptive }}(i-1)-S S(i)\right]+S S(i)}{\sum_{10}^{i} \mathrm{H}\left[T H_{\text {adaptive }}(i-1)-S S(i)\right]}
\end{gathered}
$$

where $\theta=10$ is a constant threshold, $\delta=0.1$ is the significance assigned to $i$ th $S S$ for computing the adaptive threshold $T H_{\text {adaptive }}(i)$ at the $i$ th snore and $\mathrm{H}[\beta]$ is the Heaviside step function, whose value is 0 for $\beta<0$ and 1 for $\beta>=0$.

The median threshold, $T H_{\text {median }}$, is defined as follows:

$$
\begin{gathered}
S S \_10=\{S S(i) \mid S S(i)<10\} \\
T H_{\text {median }}=\operatorname{Median}\left(S S \_10\right)+\operatorname{Std}\left(S S \_10\right)
\end{gathered}
$$

Let $R \_S S(i)$ be the time interval between successive regular snores:

$$
\begin{array}{c|l}
R_{-} S S_{\text {adaptive }}(i)=S S(i) & S S(i)<T H_{\text {adaptive }}(i) \\
R_{-} S S S_{\text {median }}(i)=S S(i) & S S(i)<T H_{\text {median }}
\end{array}
$$

Let $A \_$Snores, $R \_$Snores and NR_Snores be all snores, the selected regular snores and non-regular snores, respectively:

$$
\begin{aligned}
& A_{-} \text {Snores }=\{S(i)\} \\
& R \_ \text {Snores } \text { adaptive }=\left\{S(i) \mid R \_S S_{\text {adaptive }}(i)\right\} \\
& N R \_ \text {Snores } s_{\text {adaptive }}=\left\{S(i) \mid S(i) \notin R \_ \text {Snores } \text { adaptive }\right\}
\end{aligned}
$$

For instance, in Fig.1, $S(i+3)$ would belong to the group of NR_Snores adaptive. $_{\text {. }}$

\section{Parameters and Features of Snores}

The parameters studied were: $R \_S S(i)$, the time interval between successive regular snores, and IMean $(i)(d B$ $S P L)$, the mean snore intensity of $S(i)$. The features computed from each parameter are shown in Table II.

TABLE II

FEATURES DERIVED FROM EACH PARAMETER

\begin{tabular}{ll}
\hline \hline Name & Description \\
\hline$M$ & Mean value \\
Std & Standard Deviation \\
$C V$ & Coefficient of Variation \\
$p 25$ & $25^{\text {th }}$ percentile \\
$p 50$ & $50^{\text {th }}$ percentile \\
$p 75$ & $75^{\text {th }}$ percentile \\
Mode & Mode \\
sIQ & Semi-Interquartile Range \\
Kurt & Kurtosis of the probability distribution \\
Skew & Skewness of the probability distribution \\
Max & Maximum value \\
RMS & Root mean square \\
\hline \hline
\end{tabular}

\section{RESULTS}

We applied $T H_{\text {adaptive, }} T H_{\text {median }}$ and $T H_{10}$ to the $S S(i)$ of all 34 subjects. In the case of $T H_{\text {adaptive }}$ the $M, p 75$, Skew and $R M S$ features were significantly different in subjects with $\mathrm{AHI}<30 \mathrm{~h}^{-1}$ and in subjects with $\mathrm{AHI}>=30 \mathrm{~h}^{-1}$, with statistical significance $p<0.001$ in the Mann-Whitney $U$ test (Table IIIa). Moreover, the results were also favourable for the Std and $s I Q$ features when distinguishing between groups of subjects with AHI above and under $5 \mathrm{~h}^{-1}$ and $10 \mathrm{~h}^{-1}(\mathrm{p}<0.05)$.

For the $R \_S S(i)$ selected after applying $T H_{\text {median }}$, the features did not perform such good results (Table IIIb). The p-values obtained for all features while differentiating between subjects with AHI of 5, 10 and $15 \mathrm{~h}^{-1}$ did not have statistical significance $(\mathrm{p}>0.05)$. The only significant differences $(p<0.05)$ were obtained for seven features when comparing the groups of subjects with AHI above and under $30 \mathrm{~h}^{-1}$.

With $T_{10}$ there were no statistically significant differences observed for any feature.

To observe in what extent the $T H_{\text {adaptive }}$ offered better results, we computed the histograms of the $R \_S S_{\text {adaptive }}$ for all 34 subjects. The average of the histogram envelopes were calculated and drawn for two groups with different levels of severity: $\mathrm{AHI}<15 \mathrm{~h}^{-1}$ (Fig.2a) and $\mathrm{AHI}<30 \mathrm{~h}^{-1}$ (Fig.2b). 
TABLE III

a) Features for $R \quad S S_{\text {adaptive }}(i)$

\begin{tabular}{|c|c|c|c|c|c|c|c|c|c|c|c|c|}
\hline & & $\mathrm{M}$ & Std & $\mathrm{CV}$ & p25 & $\mathrm{p} 50$ & p75 & Mode & sIQ & Kurt & Skew & RMS \\
\hline p_5 & & 0.2525 & 0.0402 & 0.3799 & 0.3520 & 0.3799 & 0.0696 & 0.3799 & 0.0296 & 0.2307 & 0.4091 & 0.1127 \\
\hline p_10 & & 0.2157 & 0.0080 & 0.4660 & 0.3056 & 0.4162 & 0.0521 & 0.4921 & 0.0299 & 0.1129 & 0.2321 & 0.0708 \\
\hline p_15 & & 0.0632 & 0.1030 & 0.1199 & 0.1491 & 0.2225 & 0.0485 & 0.7177 & 0.2518 & 0.1112 & 0.0403 & 0.0223 \\
\hline p_30 & & 0.0096 & 0.0733 & 0.0378 & 0.0179 & 0.0316 & 0.0096 & 0.8253 & 0.1501 & 0.0289 & 0.0077 & 0.0043 \\
\hline \multirow{2}{*}{$\mathbf{G} \mathbf{A H I}<\mathbf{3 0}$} & $\mathrm{m}$ & 1.853 & 0.919 & 0.772 & 1.406 & 1.825 & 2.271 & 0.844 & 0.432 & 19.545 & 1.062 & 2.146 \\
\hline & $\mathrm{s}$ & 0.861 & 0.311 & 0.699 & 1.086 & 1.049 & 1.089 & 1.188 & 0.397 & 21.394 & 2.488 & 0.696 \\
\hline \multirow{2}{*}{ G AHI $>=30$} & $\mathrm{~m}$ & 0.918 & 0.701 & 1.416 & 0.651 & 0.810 & 1.042 & 0.585 & 0.196 & 55.814 & 4.777 & 1.240 \\
\hline & $\mathrm{s}$ & 0.898 & 0.301 & 0.812 & 0.943 & 1.040 & 1.060 & 0.972 & 0.126 & 70.144 & 4.475 & 0.826 \\
\hline
\end{tabular}

b) Features for $R_{-} S S_{\text {median }}(i)$

\begin{tabular}{|c|c|c|c|c|c|c|c|c|c|c|c|c|}
\hline & & $\mathrm{M}$ & Std & $\mathrm{CV}$ & $\mathrm{p} 25$ & p50 & p75 & Mode & SIQ & Kurt & Skew & RMS \\
\hline p_30 & & 0.0240 & 0.2902 & 0.0146 & 0.0162 & 0.0450 & 0.0854 & 0.8385 & 0.0346 & 0.0240 & 0.0179 & 0.0413 \\
\hline \multirow{2}{*}{$\mathbf{G} \mathbf{A H I}<\mathbf{3 0}$} & $\mathrm{m}$ & 2.564 & 0.904 & 0.361 & 2.155 & 2.712 & 3.106 & 1.145 & 0.476 & 5.633 & -1.067 & 2.736 \\
\hline & $\mathrm{s}$ & 0.405 & 0.307 & 0.140 & 0.615 & 0.414 & 0.459 & 1.297 & 0.318 & 3.156 & 0.712 & 0.390 \\
\hline \multirow{2}{*}{$\mathbf{G} \mathbf{A H I}>=\mathbf{3 0}$} & $\mathrm{m}$ & 2.083 & 0.991 & 0.533 & 1.443 & 2.192 & 2.762 & 0.905 & 0.659 & 3.361 & -0.390 & 2.331 \\
\hline & $\mathrm{s}$ & 0.727 & 0.316 & 0.224 & 0.872 & 0.869 & 0.816 & 1.223 & 0.298 & 1.913 & 0.616 & 0.713 \\
\hline
\end{tabular}

c) Features for Imean of $N R \_$Snores adaptive $_{\text {(i) }}$

\begin{tabular}{|c|c|c|c|c|c|c|c|c|c|c|c|c|c|}
\hline & & $\bar{M}$ & Std & $\mathrm{CV}$ & $\mathrm{p} 25$ & p50 & p75 & Mode & SIQ & Kurt & Skew & Max & RMS \\
\hline \multirow{2}{*}{$\begin{array}{l}\text { p_5 } \\
\text { p_10 }\end{array}$} & & 0.0100 & 0.0892 & 0.5371 & 0.0017 & 0.0100 & 0.0149 & 0.3520 & 0.0789 & 0.0789 & 0.0179 & 0.0696 & 0.0149 \\
\hline & & 0.0521 & 0.0336 & 0.2002 & 0.0265 & 0.0469 & 0.0376 & 0.4921 & 0.0299 & 0.0235 & 0.0420 & 0.0469 & 0.0640 \\
\hline \multirow{2}{*}{\multicolumn{2}{|c|}{$\begin{array}{l}\text { p_15 } \\
\text { p_30 }\end{array}$}} & 0.0530 & 0.0064 & 0.0146 & 0.0530 & 0.0632 & 0.0367 & 0.8143 & 0.0044 & 0.0044 & 0.0632 & 0.0302 & 0.0579 \\
\hline & & 0.0532 & 0.0026 & 0.0038 & 0.0578 & 0.0532 & 0.0378 & 0.7598 & 0.0007 & 0.0020 & 0.0792 & 0.0240 & 0.0532 \\
\hline \multirow{2}{*}{$\mathbf{G} \mathbf{A H I}<\mathbf{3 0}$} & $\mathrm{m}$ & 50.753 & 8.147 & 0.159 & 44.584 & 50.481 & 56.466 & 43.531 & 5.941 & 3.099 & 0.319 & 74.637 & 51.415 \\
\hline & $\mathrm{s}$ & 7.175 & 1.967 & 0.024 & 6.813 & 8.744 & 9.092 & 9.374 & 2.056 & 1.292 & 0.729 & 6.406 & 7.341 \\
\hline \multirow{2}{*}{ G AHI $>=30$} & $\mathrm{~m}$ & 54.937 & 10.278 & 0.186 & 46.299 & 55.255 & 63.205 & 44.367 & 8.453 & 2.249 & 0.038 & 78.761 & 55.906 \\
\hline & $\mathrm{s}$ & 4.443 & 1.980 & 0.027 & 3.161 & 5.370 & 6.431 & 10.462 & 2.202 & 0.773 & 0.417 & 5.769 & 4.660 \\
\hline
\end{tabular}

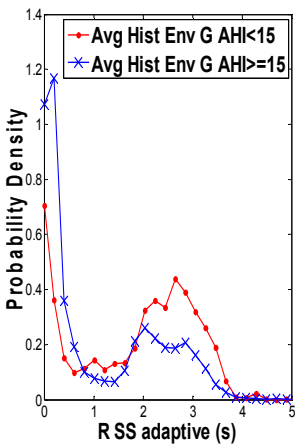

a)

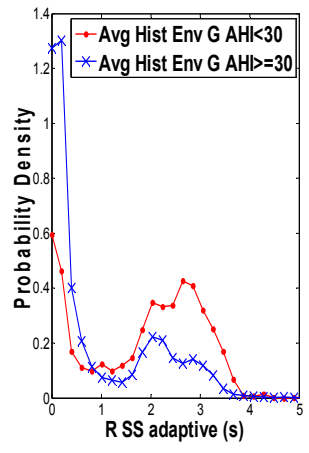

b)
Fig. 2 Average histogram envelopes of $R \_S S_{\text {adaptive }}$ a) for subjects with AHI above and under $\left.15 h^{-1} b\right)$ for subjects with AHI above and under $30 \mathrm{~h}^{-1}$.
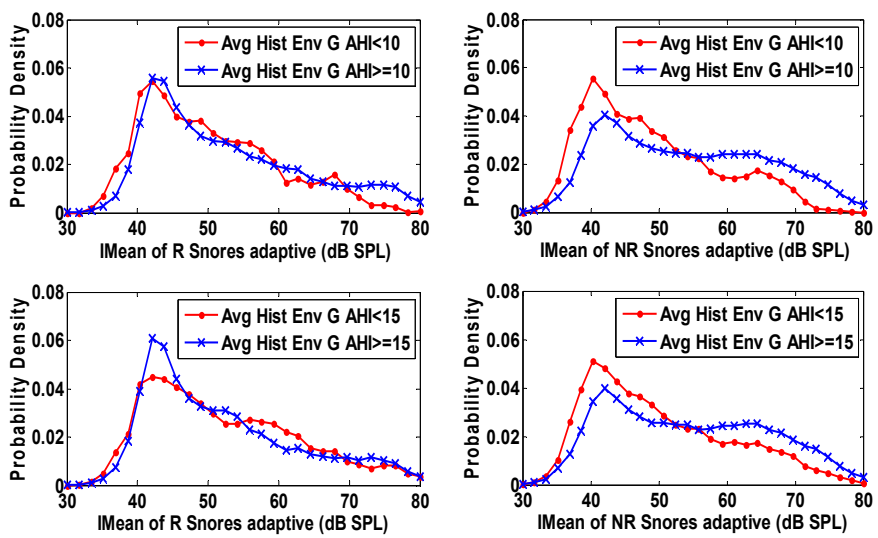

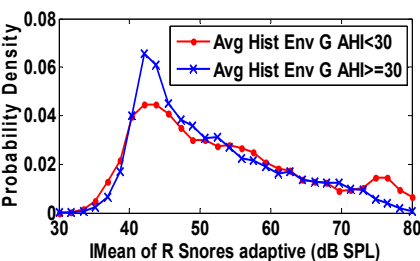

a)

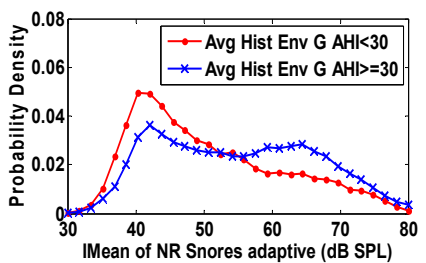

b)
Fig. 3 Average histogram envelopes for IMean of a) $R \_$Snores adaptive $_{\text {and }}$ b)

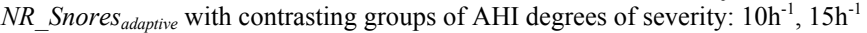
and $30 \mathrm{~h}^{-1}$. 
We can observe a clear distinction between the shape of the envelopes of subjects with AHI above and under $15 \mathrm{~h}^{-1}$ and $30 \mathrm{~h}^{-1}$, which confirm the p-values obtained. Moreover, we can observe that the number of $R \_S S$ events under 1 second is much higher for subjects with AHI greater than $15 \mathrm{~h}^{-1}$ or $30 \mathrm{~h}^{-1}$. This indicates that most severe patients tend to snore in consecutive breathing events, i.e. they snore in consecutive inspiration and exhalation events.

Since $T H_{\text {adaptive }}$ performed better than $T H_{\text {median }}$ we decided to analyze quantitatively the mean intensity of $R \_$Snores adaptive $_{\text {and }}$ NR_Snores adaptive $_{\text {. }}$

In the case of $R$ Snores adaptive there were no Imean features for which the $\mathrm{p}$-value was under 0.05 significance level. On the other hand, there are 6 Imean features derived from non-regular snores that show high statistically significant differences $(p<0.001)$ between subjects from contrasting groups of AHI severity (Table IIIc).

We computed the Imean histograms for the regular and non-regular snores (Fig.3).

With respect to regular snores (Fig.3a) the shape of the histograms is very similar for the two groups with contrasting AHI. Conversely, the results obtained for nonregular snores (Fig.3b) show utterly different shapes for the two groups of subjects. All 3 plots shown on Fig.3b make evidence that non-regular snores from subjects with higher AHI have a mean intensity predominantly above $55 \mathrm{~dB}$ SPL with respect to subjects of lower AHI.

\section{CONCLUSIONS}

The application of two distinctly mathematically structured thresholds to the time interval between successive snores of 34 subjects was essential to properly select regular snores from all-night snore episode sequences. $T H_{\text {adaptive }}$ outperformed $T H_{\text {median }}$ on that purpose which is intuitively explained since the former takes into account the evolution of $S S(i)$ through all night sleep as it is based on the concept of an adaptive estimation.

We observe that the time interval between successive regular snores has distinct distribution for subjects with high and low levels of AHI. The $R \_S S$ events under one second are much more frequent for subjects with high AHI index since they have propensity to snore in consecutive inspiration and exhalation events. In addition, several $R \_S S_{\text {adaptive }}$ features allowed the statistical distinction of subjects with high and low levels of AHI.

Regular snores have very similar mean intensity values for all 4 levels of AHI severity considered: $\mathrm{AHI}<5 \mathrm{~h}^{-1}$, $\mathrm{AHI}<10 \mathrm{~h}^{-1}, \mathrm{AHI}<15 \mathrm{~h}^{-1}$ and $\mathrm{AHI}<30 \mathrm{~h}^{-1}$. On the other hand, in the case of non-regular snores we can observe very different shapes on the mean intensity histograms for subjects with opposite AHI degrees of severity, which were confirmed by statistically significant differences between populations. Furthermore, we found that nonregular snores from the most severe SAHS subjects present very frequently mean intensity values above $55 \mathrm{~dB}$ SPL with respect to subjects of lower AHI.

This makes evidence that non regular snores carry a large extent of information with respect to the screening of SAHS based on sound intensity.

Once we classify, from a whole sequence of snores, the ones that are regular and the ones that are non-regular, we can study these two groups of snores separately and explore its potential to screen subjects with SAHS.

The concept of regular snores is a new and promising approach to study the snore mechanism production and can help evaluating the performance of already available techniques being applied to snoring.

\section{REFERENCES}

[1] J. Durán-Cantolla, J. Mar, G. Torre Muñecas, R. Rubio Aramendi, and L. Guerra, "The Availability in Spanish Public Hospitals of Resources for Diagnosing and Treating Sleep Apnea-Hypopnea Syndrome," Archivos de Bronconeumologia, vol. 40, no. 6, pp. 259267, June2004.

[2] D. Pevernagie, R. M. Aarts, and M. De Meyer, "The acoustics of snoring," Sleep Medicine Reviews, vol. 14, no. 2, pp. 131-144, Apr.2010.

[3] U. Abeyratne, A. Karunajeewa, and C. Hukins, "Mixed-phase modeling in snore sound analysis," Medical and Biological Engineering and Computing, vol. 45, no. 8, pp. 791-806, Aug.2007.

[4] A. Ng, T. Koh, U. Abeyratne, and K. Puvanendran, "Investigation of Obstructive Sleep Apnea Using Nonlinear Mode Interactions in Nonstationary Snore Signals," Annals of Biomedical Engineering, vol. 37, no. 9, pp. 1796-1806, Sept.2009.

[5] J. A. Fiz, R. Jané, J. Solà-Soler, J. Abad, M. Á. García, and J. Morera, "Continuous analysis and monitoring of snores and their relationship to the apnea-hypopnea index," The Laryngoscope, vol. 120, no. 4, pp. 854-862, 2010.

[6] M.Cavusoglu et al., "Investigation of sequential properties of snoring episodes for obstructive sleep apnoea identification," Physiological Measurement, vol. 29, no. 8, pp. 879-898, 2008.

[7] "Sleep-related breathing disorders in adults: recommendations for syndrome definition and measurement techniques in clinical research. The Report of an American Academy of Sleep Medicine Task force," Sleep 1999,22,667-689, Aug.1999.

[8] "Practice parameters for the indications for polysomnography and related procedures:an update for 2005. The Report of an American Academy of Sleep Medicine Task force," Sleep 2005,28(4),499-521, Apr.2005.

[9] R. Jané, J. A. Fiz, J. Solà-Soler, S. Blanch, P. Artís, and J. Morera, "Automatic snoring signal analysis in sleep studies," Proceedings of the 25th Annual International Conference of the IEEE, 2003, pp. 366-369.

[10] J. Solà-Soler, R. Jané, J. A. Fiz, and J. Morera, "Variability of snore parameters in time and frequency domains in snoring subjects with and without Obstructive Sleep Apnea," Proceedings of the 27th Annual International Conference of the IEEE, 2005, pp. 2583-2586. 